\title{
Hopf-Fold Bifurcation Analysis in a delayed Gause-Type Predator-Prey Models
}

\author{
Shuang Guo ${ }^{1}$, Xiuli Li, Jian Yu and Xiu Ren \\ Department of Mathematics, Teacher Education Institute, Daqing Normal \\ University, Daqing 163712, China \\ 'guofeixue721030@163.com
}

\begin{abstract}
In this paper, we analyzed the distribution of the roots of the associated characteristic equation for the Gause type predator-prey model. The point of bifurcation and a group of conditions of existence of Hopf-Fold bifurcation were obtained at the coexisting equilibrium. There are complex dynamic phenomenons such as periodic motion, quasi--periodic motion and bursting behavior by the numerical simulations.
\end{abstract}

Keywords: predator-prey models; delay; Hopf-Fold bifurcation; bursting behavior

\section{Introduction}

The importance of the relationship between predator and prey has been widely studied in pure ecology and in mathematical biology. It has the important signifycance in protecting the resources and environment, maintaining the balance of the ecosystem and reasonable use of biological resources and so on. In 1977, Freedman and Waltman [1] gaved a class of Gause-tape food chain model:

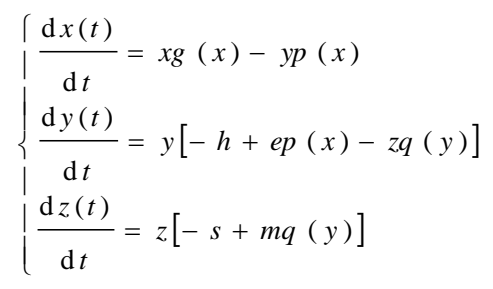

where $x(t), y(t)$ and $z(t)$ are the population densities of prey, predator and top predator at time $t$, respectively. $g(x)$ is the intrinsic growth rate of prey; $p(x)$ and $q(y)$ are the specific growth rates of predator and top predator; $h, s>0$ are the death rates of predator and top predator; $e, m>0$ are the conversion rates for prey and predator. Many authors established the stability criteria and argued that the unique interior equilibrium exists and is locally asymptotically stable. See [2-8]. Ginoux [9] highlighted this model has several Hopf bifurcations and a period-doubling cascade generating a snail shell-shaped chaotic attractor. Hastings and Powell [10] discussed a continuous time model of a food chain incorporating nonlinear functional responses, and the model exhibits chaotic in long-term behavior when appropriate biologically reasonable parameter values are chosen. In [11], the eigenvalue problem for the linearized system at the coexisting equilibrium and a group of sufficient conditions for the existence of Hopf bifurcation are obtained. The direction of the Hopf bifurcation and the stability of the bifurcated periodic solutions are determined by applying the normal form method and the center manifold theorem. But with the further changes of delay, the occurrence of quasi-periodic motion by some numerical simulations and even bursting behavior.

In nature, the population will generally experience some periodic oscillation. It can be 
regarded as by the impact of delay from a mathematical point. See [12-19]. For this model, the purpose of current work is to analysis the effect of delay on the dynamics. We choose

$$
g(x)=\alpha\left(1-\frac{x}{K}\right), \quad p(x)=\frac{\beta x}{1+p x}, q(y)=r y,
$$

where $\alpha, \beta, K, p, r$ are positive parameters. So the model is the following form:

$$
\left\{\begin{array}{l}
\frac{\mathrm{d} x(t)}{\mathrm{d} t}=\alpha x\left(1-\frac{x}{K}\right)-\frac{\beta y x}{1+p x} \\
\frac{\mathrm{d} y(t)}{\mathrm{d} t}=y\left(-l+\frac{e \beta x(t-\tau)}{1+p x(t-\tau)}-r z\right) \\
\frac{\mathrm{d} z(t)}{\mathrm{d} t}=z(-s+m r y(t-\tau))
\end{array}\right.
$$

In this paper, we still let $\tau$ as the bifurcation parameter and consider the delay Gause-type predator-prey model.

The rest of our paper is organized as follows: in Section 2, the case of a zero and a pair of purely imaginary eigenvalues for the linearization of Eq.(1.2) at the coexisting equilibrium $\bar{E}\left(x^{*}, y^{*}, z^{*}\right)$ is addressed. In Section 3, we perform the center manifold reduction and normal for computation. In Section 4, we carry out some numerical simulations and bifurcation analysis to illustrate the results obtained. Finally, the conclusion is obtained in Section 5.

\section{Hopf-Fold Bifurcation}

For the sake of convenience, we non-dimensionalizes the Eq.(1.2), then the Eq.(1.2) takes the form:

$$
\left\{\begin{array}{l}
\frac{\mathrm{d} x(t)}{\mathrm{d} t}=x(1-x)-a y \frac{x}{b+x} \\
\frac{\mathrm{dy}(t)}{\mathrm{d} t}=y\left(-l+\frac{c x(t-\tau)}{b+x(t-\tau)}-r z\right) \\
\frac{\mathrm{d} z(t)}{\mathrm{d} t}=z(-s+d y(t-\tau))
\end{array}\right.
$$

which satisfies

$$
x_{0}(\theta)=\phi_{1}(\theta) \geq 0
$$

$y_{0}(\theta)=\phi_{2}(\theta) \geq 0, z_{0}(\theta)=\phi_{3}(\theta) \geq 0, \theta \in[-\tau, 0]$,

$x(0)>0, y(0)>0, z(0)>0, \phi(\theta)=\left(\phi_{1}, \phi_{2}, \phi_{3}\right) \in C\left([-1,0], R^{3}\right)$,

$\|\phi\|=\max \{|\phi(\theta)|: \theta \in[-\tau, 0]\}$, and $|\phi|$ is any norm in $R^{3}$.

Where

$$
a=\frac{\beta}{p K \alpha}, b=\frac{1}{p K}, l=\frac{h}{\alpha}, c=\frac{e \beta}{p \alpha}, d=m r .
$$

Obviously, the delay can't change the number of equilibria and non-dimensionalizes can't change the properties of system. In the following, we always assume Eq.(2.1) has a positive equilibrium exists and denote it by $\bar{E}\left(x^{*}, y^{*}, z^{*}\right)$ with

$$
x^{*}=\frac{(1-b)+\sqrt{(1-b)^{2}+4 b-4 a s / d}}{2}, \quad y^{*}=\frac{s}{d}, \quad z^{*}=\frac{c x *}{r(x *+b)}-\frac{l}{r}
$$

We consider the linearized system of $(2.1)$ at $\bar{E}$. The Jacobian determinant is the following: 


$$
\left|\begin{array}{ccc}
\lambda-m_{11} & -m_{12} & 0 \\
-n_{21} e^{-\lambda \tau} & \lambda & -m_{23} \\
0 & -n_{32} e^{-\lambda \tau} & \lambda
\end{array}\right|
$$

Where $m_{11}=1-2 x^{*}-\frac{a b y *}{\left(b+x^{*}\right)^{2}}, m_{12}=-\frac{a x^{*}}{b+x^{*}}<0, m_{23}=-r y *<0$,

$$
m_{32}=d z *>0, n_{21}=-\frac{b c y *}{(b+x *)^{2}}<0 .
$$

The characteristic equation at $\bar{E}$ is given by

$D(\lambda, \tau)=\lambda^{3}+a_{2} \lambda^{2}+\left(b_{1} \lambda+b_{0}\right) e^{-\lambda \tau}=0$,

where $a_{2}=-m_{11}, b_{1}=-m_{12} n_{21}-m_{23} n_{32}>0$ and $b_{0}=m_{11} m_{23} n_{32}$.

If $m_{11}<0$, the model (1.2) will appear Hopf bifurcation. We let $m_{11}=0$, then $a_{2}=0, b_{0}=0$ and $b_{1}>0$. We take a derivative with respect to Eq.(2.2), we have

$$
\left.\frac{\mathrm{d} D(\lambda, \tau)}{\mathrm{d} \lambda}\right|_{\lambda=0}=b_{1} \neq 0 .
$$

This means $\lambda=0$ is the simple root of= Eq.(2.2) when $m_{11}=0$.

Now we substitute $\lambda=i \omega(\omega>0)$ into Eq.(2.2), separating the real and imaginary parts gives

$\omega^{3}-b_{1} \omega \cos \omega \tau=0, \quad b_{1} \sin \omega \tau=0$

Simplify (2.3), we have

$\omega^{4}-b_{1}{ }^{2}=0$

Eq.(2.4) exists the positive root which satisfied $\omega_{0}{ }^{2}=b_{1}$, from equation (2.3) and (2.4), we have

$$
\tau_{k}=\frac{(2 k+2) \pi}{\omega_{0}}, \quad k=0, \quad 1, \quad 2, \ldots \ldots
$$

Denoting $\lambda(\tau)=\alpha(\tau)+i \omega(\tau)$ be the root of Eq.(2.3) satisfying $\alpha\left(\tau_{0}\right)=0$, $\omega\left(\tau_{0}\right)=\omega_{0}$, denote $q^{*}=\frac{\left(1-x^{*}\right)^{2}}{1-2 x^{*}}\left(\Leftrightarrow m_{11}=0\right)$, then we have the following theorem.

Theorem 1.1 Suppose $q=q *$ and $\tau=\tau_{0}$, then all the roots of Eq.(2.3), except $\lambda=0$ and $\lambda= \pm i \omega_{0}$, have negative real parts.

Proof On the basis of the foregoing we know that Eq. (2.3\}) has a single zero root and a simple pair of pure imaginary roots. Suppose that Eq. (2.3) has a root with positive real part denoted $\lambda=\alpha_{0}+i \beta_{0}$ when $q=q^{*}$ and $\tau=\tau_{0}$. Let $\lambda=\alpha(\tau)+i \beta(\tau)$ be the root of Eq.(2.3) with $q=q^{*}$ satisfying $\alpha\left(\tau_{0}\right)=\alpha_{0}>0$ and $\beta\left(\tau_{0}\right)=\beta_{0}$. Then there exists a positive number $0<\varsigma<\tau_{0}$ such that $\alpha(\tau)>0$ when $\tau \in\left(\tau_{0}-\varsigma, \tau_{0}\right)$.

Because

$$
D(\lambda, 0)=\lambda^{3}+b_{1} \lambda=0
$$

has a single zero root and two roots with negative real part when $\tau=0$. Furthermore,

$$
\left\lfloor\frac{\mathrm{d} \lambda(\tau)}{\mathrm{d} \tau}\right]^{-1}=\frac{3 \lambda^{2} e^{\lambda \tau}+b_{1}}{b_{1} \lambda^{2}}-\frac{\tau}{\lambda}
$$


and

$$
\begin{aligned}
& \quad \operatorname{sign}\left[\frac{\mathrm{d} \operatorname{Re} \lambda(\tau)}{\mathrm{d} \tau}\right]^{-1}{ }_{\tau=\tau_{0}} \\
& \left.=\operatorname{sign} \operatorname{Re}\left[\frac{\left[\lambda^{2} e^{\lambda \tau}+b_{1}\right.}{b_{1} \lambda^{2}}\right]_{\tau=\tau_{0}}\right]_{\tau=\tau_{0}} \\
& =\operatorname{sig}\left\lceil\frac{-3 \omega_{0}{ }^{2} \cos \omega_{0} \tau+b_{1}}{-b_{1} \omega_{0}{ }^{2}} \mid \operatorname{sigh} \frac{2}{b_{1}{ }^{2}}\right\rfloor_{\tau=\tau_{0}}>0
\end{aligned}
$$

This contradicts the conclusion of suppose and completes the proof.

Then the system (2.2) can undergo a Hopf -Fold bifurcation at $\bar{E}$ when $q=q *$. For convenience, we denote the Hopf-Fold bifurcation point in the parameter plane by $\left(m_{11}, \tau\right)$ by $\left(0, \tau_{0}\right)$. We will find that there is a family of smooth three-dimensional invariant manifolds near the $\bar{E}$ and the dynamics of system (2.1) near the $\bar{E}$ can be determined by its restriction on the manifold.

\section{The Normal Form}

In general, the universal unfolding need deriveing for studying the stability of $\bar{E}$ at the critical case. Our method is based on the center manifold theory [19] and normal form method [20].

Let $x_{1}(t)=x(t)-x^{*}, x_{2}(t)=y(t)-y *, x_{3}(t)=z(t)-z^{*}, X_{i}(t)=x_{i}(\tau t)(i=1,2,3)$, $m_{11}=0+\mu_{1}$ and $\tau=\tau_{0}+\mu_{2},\left(\mu_{1}, \mu_{2}\right) \in R$, and

$$
A=\left(\begin{array}{ccc}
m_{11} & m_{12} & 0 \\
0 & 0 & m_{23} \\
0 & 0 & 0
\end{array}\right), \quad C=\left(\begin{array}{ccc}
0 & 0 & 0 \\
n_{21} & 0 & 0 \\
0 & n_{32} & 0
\end{array}\right)
$$

The system (2.1) is transformed into a functional differential equation in $C=C\left([-1,0], R^{3}\right)$ as

$\frac{\mathrm{d} x}{\mathrm{~d} t}=L_{\mu}\left(x_{t}\right)+f\left(\mu, x_{t}\right)$

where $\quad x(t)=\left(x_{1}(t), x_{2}(t), x_{3}(t)\right)^{T} \in R^{3}, \quad x_{t} \in C$ is defined by $x_{t}=x_{t}(\theta)$, $-1 \leq \theta \leq 0$,

$\mu=\left(\mu_{1}, \mu_{2}\right)^{T}$ and $L_{\mu}: C \rightarrow R, f: R \times C \rightarrow R$ are given respectively by $L_{\mu}(\phi)=\left(\tau_{0}+\mu_{2}\right) A \phi(0)+\left(\tau_{0}+\mu_{2}\right) C \phi(-1)$,

where $\phi=\left(\phi_{1}, \phi_{2}, \phi_{3}\right)^{T} \in C\left([-1,0], R^{3}\right)$.

There exists a $3 \times 3$ matrix $\eta(\theta, \mu) \quad(-1 \leq \theta \leq 0)$, whose elements are of bounded variation functions such that

$L_{\mu}(\phi)=\int_{-1}^{0}[\mathrm{~d} \eta(\theta, \mu)] \phi(\theta), \quad$ for $\phi \in C\left([-1,0], R^{3}\right)$ 
In fact, we can choose

$$
\eta(\theta, \mu)=\left\{\begin{array}{lr}
\left(\tau_{0}+\mu_{2}\right) A, & \theta=0 \\
0, & \theta \in(-1,0) \\
\left(\tau_{0}+\mu_{2}\right) C, & \theta=-1
\end{array}\right.
$$

For $\Psi \in C^{1}\left([-1,0], R^{3}\right)$ and $\varphi \in C$, we define a bilinear form

$$
\langle\psi, \phi\rangle=\psi(0) \phi(0)-\int_{-1}^{0} \int_{\varsigma=0}^{\theta} \psi(\varsigma-\theta) \mathrm{d} \eta(\theta) \phi(\varsigma) \mathrm{d} \xi .
$$

Then the phase space $C$ is decomposed by $\wedge=\left\{0, \pm i \omega_{0} \tau_{0}\right\}$ as $C=P \oplus Q$, where $Q=\left\{\varphi \in C:(\psi, \varphi)=0\right.$, for all $\left.\psi \in P^{*}\right\}$, and the bases for $P$ and its adjoint $P *$ can be canculated.

It's easy to verify that $(\Psi, \Phi)=I$, the dual bases satisfy $\dot{\Phi}=\Phi B$ and $\dot{\Psi}=-B \Psi$, where

$$
B=\left\{\begin{array}{ccc}
0 & 0 & 0 \\
0 & i \omega_{0} \tau_{0} & 0 \\
0 & 0 & -i \omega_{0} \tau_{0}
\end{array}\right)
$$

As in Faria and magalhaes, we consider the enlarged phase space $B C$ of functions from $[-1,0)$ to $R^{3}$, continuous on $[-1,0)$, and with a possible jump discontinuity at zero. This space can be identified with $C \times R^{3}$. Thus its elements are writen in the form $\phi=\varphi+X_{0} c$, where $\varphi \in C, c \in R^{2}$ and $X_{0}$ is the $3 \times 3$ matrix-valued function defined by $X_{0}=0$ for $\theta \in[-1,0)$ and $X_{0}(0)=I$. In $B C$, Eq.(2.1) becomes an abstract ODE

$$
\frac{\mathrm{d}}{\mathrm{d} t} \dot{v}=A v+X_{0} G(v, \mu)
$$

where $v \in C$, and $A$ is an extension of the infinitesimal generator $A_{0}$, defined by

$$
A: C^{1} \rightarrow B C, A \varphi=\dot{\varphi}+X_{0}[L(0) \varphi-\dot{\varphi}(0)]
$$

and $G(\varphi, \mu)=(L(\mu)-L(0)) \varphi+F(\varphi, \mu)$.

Define the continuous projection $\pi: B C \rightarrow P$, $\pi\left(\varphi+X_{0} c\right)=\Phi[(\Psi, \varphi)+\Psi(0) c]$, then we can decompose the enlarged phase space by $\wedge$. as $B C=P \oplus \operatorname{Ker} \pi$, the abstract ODE is therefore decomposed as the system

$\left\{\begin{array}{l}\dot{z}=B z+\Psi(0) G(\Phi z+y, \mu) \\ \dot{y}=A_{Q}^{1} y+(I-\pi) X_{0} G(\Phi z+y, \mu)\end{array}\right.$

for $z \in R^{3}, y \in Q^{1}:=Q \cap C^{1} \subset \operatorname{Ker} \pi$, where $A_{Q}^{1}$ is the restriction of $\mathrm{A}$ as an operator from $Q^{1}$ to the Banach space $\mathrm{Ker} \pi$.

Let $M_{2}$ denote the operator defined in $V_{2}^{6}\left(C^{3} \times \operatorname{Ker} \pi\right)$, with

$$
M_{2}^{1}: V_{2}^{6}\left(C^{3}\right) \rightarrow V_{2}^{6}\left(C^{3}\right), \quad M_{2}^{1}(p)(z, \mu)=D_{z} p(z, \mu) B z-B p(z, \mu) .
$$

Then it is easy to check that one many choose the decomposition

$$
V_{2}^{6}\left(C^{3}\right)=\operatorname{Im}\left(M_{2}^{1}\right) \oplus \operatorname{Im}\left(M_{2}^{1}\right)^{c}
$$


with complementary space $\operatorname{Im}\left(M_{2}^{1}\right)^{c}$ spanned by the elements

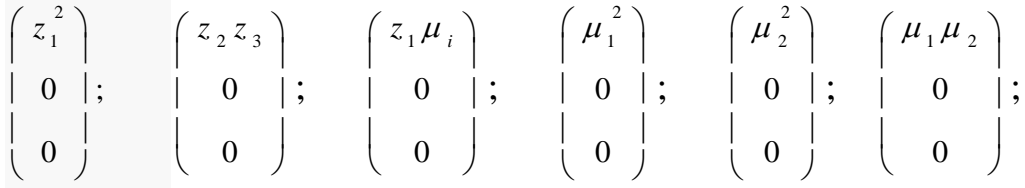

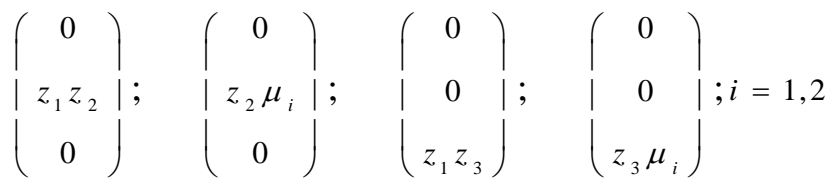

According to [13], the normal form of Eq.(2.1) on the center manifold of the origin near $\mu=0$ has the form

$\dot{z}=B z+\frac{1}{2 !} g_{2}^{1}(z, 0, \mu)+\cdots \cdots$

where $z=\left(z_{1}, z_{2}, z_{3}\right)^{T} \in R^{3}$, and $g_{2}^{1}(z, 0, \mu)$ is the function giving the quadratic terms in $(z, \mu)$ for $y=0$, which is determined by $\left.g_{2}^{1}(z, 0, \mu)=\operatorname{Pr} \operatorname{ojm}\left(M_{2}^{1}\right)^{c} \circ f_{2}^{1}(z, 0, \mu)\right)$.

Make the changes of variables:

$$
z_{1}=z, \quad z_{2}=\rho \cos \theta-\rho \sin \theta, \quad z_{3}=\rho \cos \theta+\rho \sin \theta
$$

Then Eq.(2.1) can be written in cylindrical coordinates $(\rho, z, \theta)$, and the equations of $\rho$ and $z$ are independent of the equation of $\theta$. Thus we only need to study the plannar system for $(\rho, z)$ and truncated it to the second order.

$\left\{\begin{array}{l}\dot{\rho}=\varepsilon_{1} \rho+a \rho z \\ \dot{z}=\varepsilon_{2} z+b \rho^{2}-z^{2}\end{array}\right.$

where $\varepsilon_{1}, \varepsilon_{2}, a, b$ can calculate. There are four different topological structure depending on the signs of $a$ and $b$ (See [13]). We know that stationary solutions on the z-axis of the planar system (3.6) are associated with equilibria of Eq. (2.1), and stationary solution on this axis corresponds to a periodic solution. The phase portraits and bifurcation diagram will be discussed in Section 4.

\section{Numerical Simulations and Discussions}

We choose a set of parameters:

$$
a=0.22, b=0.3, c=0.532, r=0.22, s=0.15, d=0.55, l=0.22 \text {, }
$$

Through a simple calculation, we have $q^{*}=2.578, \tau_{0}=0.1667$, we choose four different sets of parameter values $(q, \tau)=(1.442,0.025),(1.985,0.175)$, $(2,6978,0.265)$ and $(5.017,0.465)$ which corresponds to the different parameter curves Figure 1-Figure 4.

The coexistence equilibrium will ultimately to stability after a period of fluctuation in Figure 1. A stable periodic solution appears near the equilibrium with the increase of time delay in Figure 2. The number of populations appears the quasi periodic change in Figure 3 , cycle gradually increases and it will has a tendency to burst. The explosion and extinction of population will happen in Figure 4, it may lead to the collapse of the ecological system. These complex dynamics Learning behavior conform to the change 
law of population in the ecosystem. Species are in danger of extinction because of the population numbers have reached the maximum or minimum. It has the important theoretical and practical significance in studying the changing rule of the population.
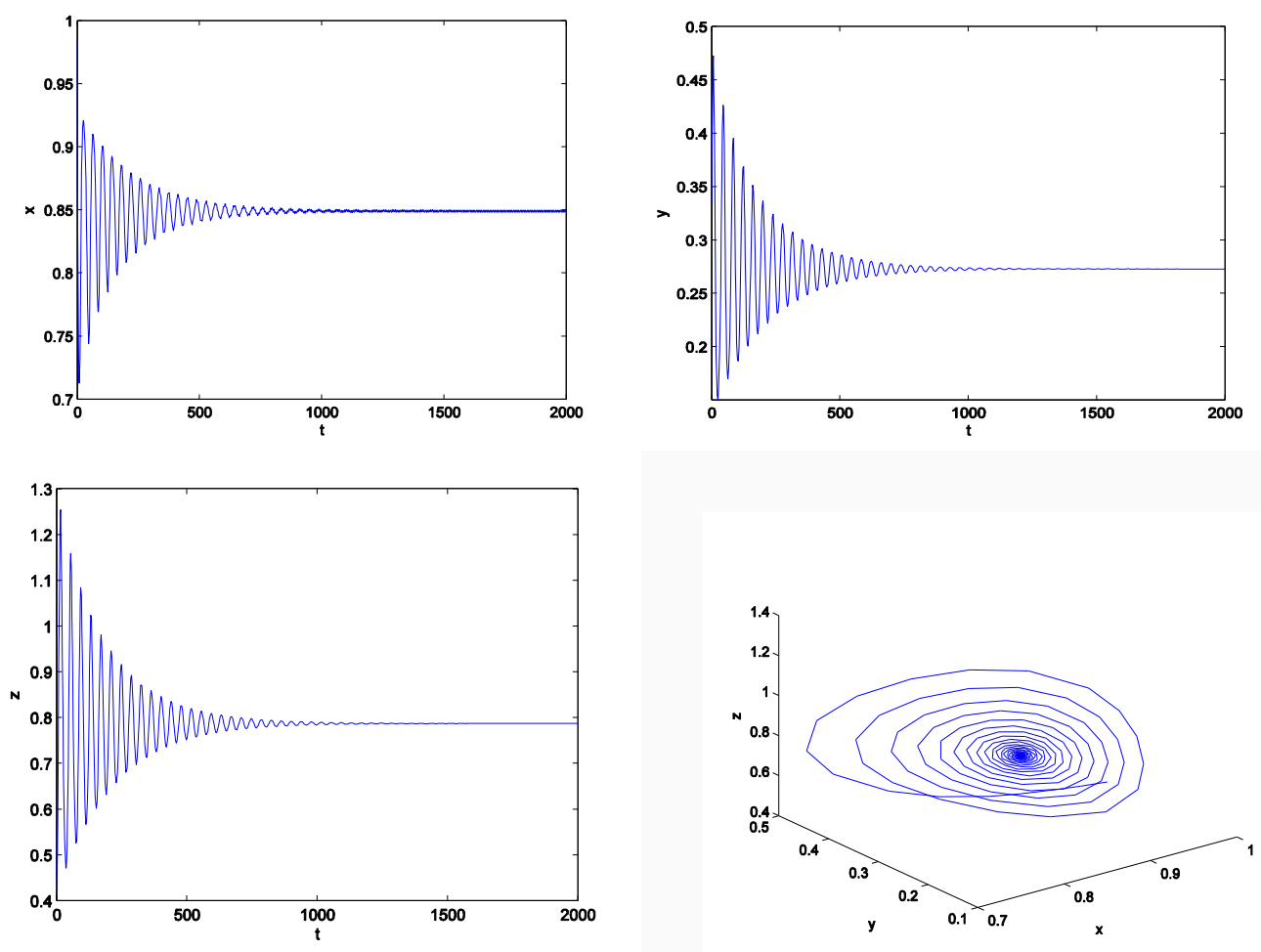

Figure 1. $\bar{E}$ is Asymptotically Stable When $q=1.442, \tau=0.025$
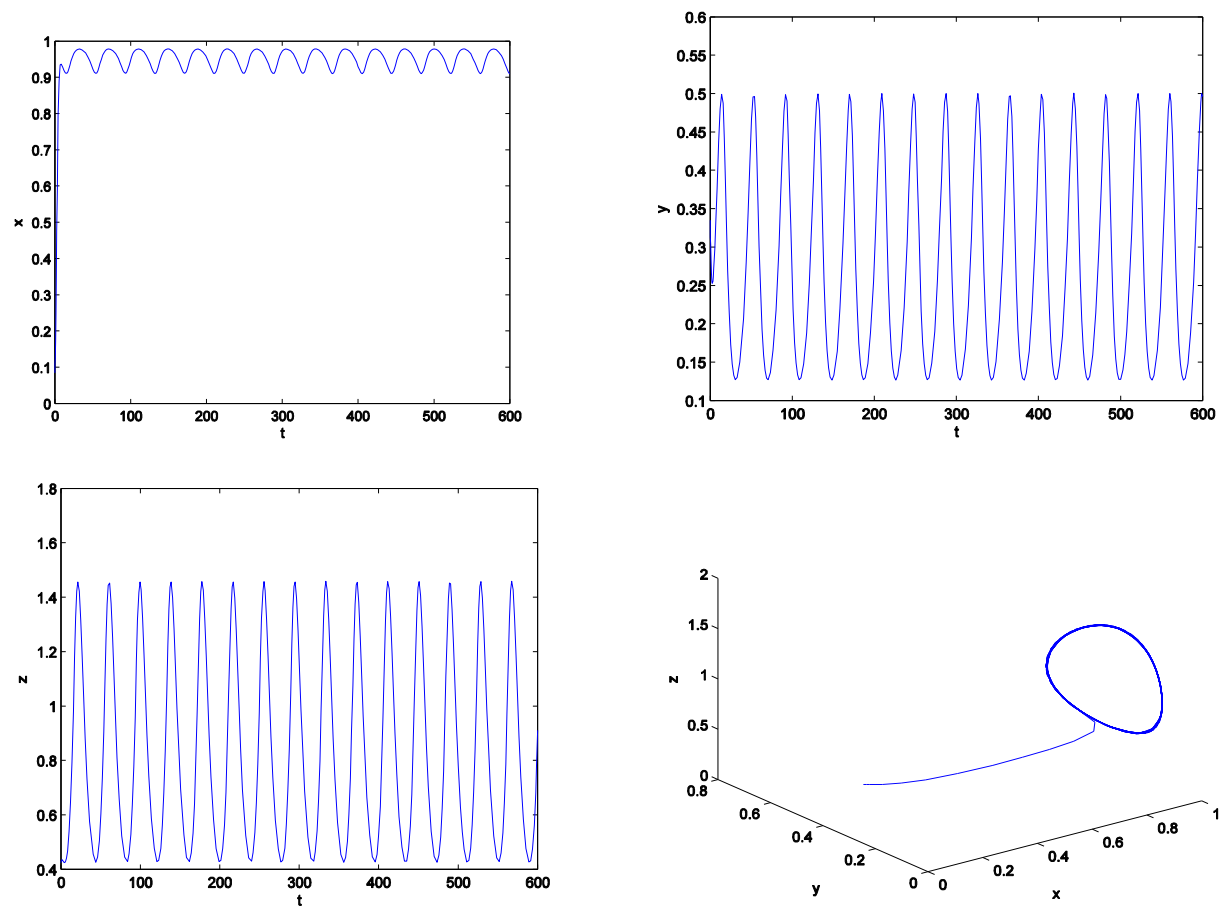

Figure 2. The Periodic Motions Near $\bar{E}$ when $q=1.985, \tau=0.175$ 

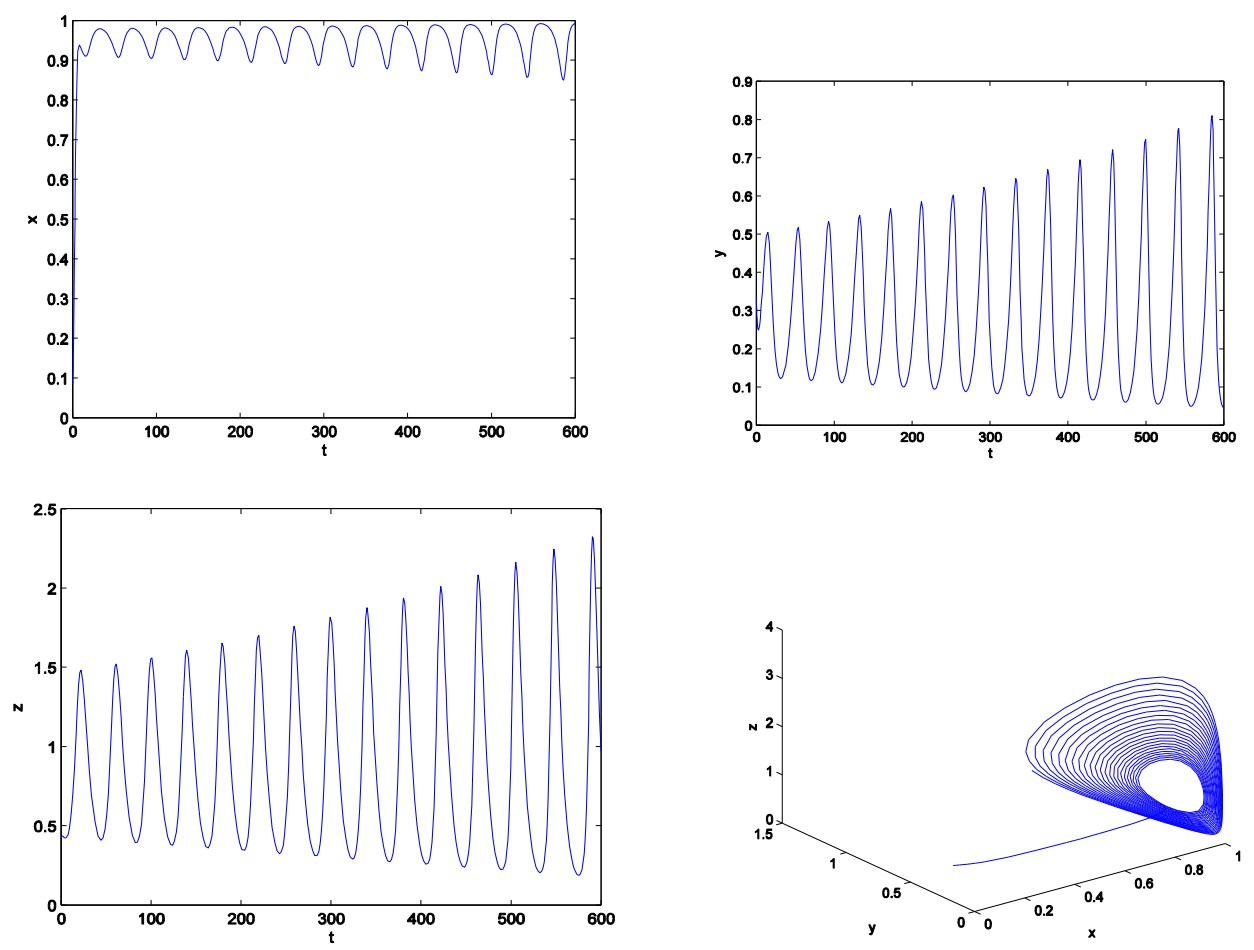

Figure 3. The Quasi-Periodic Motion Near $\bar{E}$ when $q=2.6978, \tau=0.265$
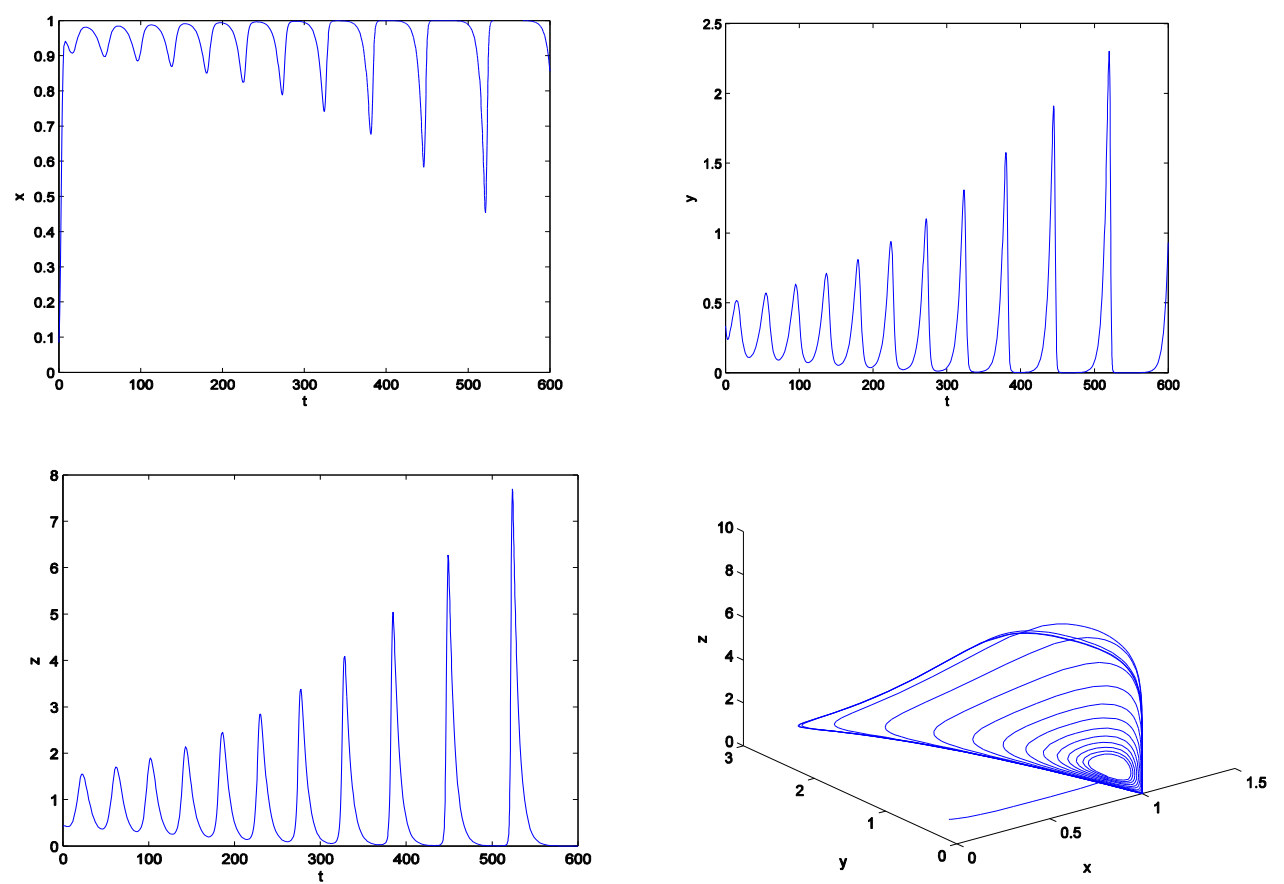

Figure 4. The Bursting Behavior Near $\bar{E}$ when $q=5.017, \tau=0.465$

\section{Conclusion}

In this paper, we investigate the Hopf-Fold bifurcation in a delayed Gause-type predator-prey model by employing the center manifold theory and normal form method. 
We have derived stability and bifurcation of the zero solution near the critical value. We show that the dynamics of system (2.1) near the Hopf-Fold point $\left(q^{*}, \tau_{0}\right)$ depends on the time delay. Under the different delays, complex dynamics, such as the quasi-periodic motion, bursting behavior can be observed. Our investigation shows that the oscillating modes in system (2.1) largely depend on the time delay.

\section{Acknowledgments}

The work is supported by Heilongjiang Provincial Natural Science Foundation (No. A201313) and the PhD Start-up Fund of Daqing Normal University (No. 14ZR09).

\section{References}

[1] H. I. Freedman and P. Waltman, "Math. Biosci", vol. 33, no. 3, (1977).

[2] S. B. Hsu and H. Wang, T. W. J. "Math", vol. 3, no. 1, (1999).

[3] G. R. Liu, W.P. Yan and J. R. Yan, "Nonlinear Anal-Theor", vol. 71, no.10, (2009).

[4] L. J. Chen and F. D Chen, "International Journal of Biomathematics", vol. 3, no. 2, (2010).

[5] D. M. Xiao, W. X. Li and P. Edinburgh, "Math. Soc", vol. 1, no. 46, (2003).

[6] Y. Kuang, E. Beretta and J. Biol, "Math", vol. 36, no. 10, (1998).

[7] S. Sarwardi, M. Haque and P. K. Mandal, "Nonlinear Dynam", vol. 3, no. 69, (2012).

[8] E. Beretta and Y. Kuang, "Nonlinear AnaL-Theor.", vol. 32, no. 3, (1998).

[9] J. M. Ginoux, B. Rossetto and J. L. Jamet, Int. J. Bifurcat, Chaos, vol. 15, no. 5, (2005).

[10] A. Hastings and T. Powell, "Ecology", vol. 72, no. 3, (1991).

[11] S. Guo and W. H. Jiang, J. Appl, Math, ID:260798, (2012).

[12] W. H. Jiang and Y. Yuan, "Physica D", no. 227, (2007).

[13] W. H. Jiang and H. B. Wang, "Nonlinear Analysis", no. 73, (2010).

[14] H. B. Wang and W. H. Jiang, J. Math, Anal. Appl, no. 368, (2010).

[15] Y. Kuznetsov, "Element of Applied Bifurcation Theory", Applied Mathematical Science, Springer, New York, (2004).

[16] S. N. Chow, C. Li and D. Wang, "Normal forms and bifurcation of planar vector fields", Cambridge University Press, Cambridge, (1994).

[17] W. H. Jiang, H. B. Wang and J. J. Wei, "Chaos Soliton Fract", vol. 3, no. 36, (2008).

[18] B. Zhen and J. Xu, Int. J. Bifurcat, Chaos, vol. 20, (2010).

[19] M. Haque, "Math. Biol", vol. 2, no. 71, (2009).

[20] T. Faria, and L. T. Magalhases, J. Differ, Equations, vol. 8, (1996).

\section{Author}

Shuang Guo, Female, Professor, Doctor of Science, the Teacher of the Daqing Normal University. 
International Journal of $\mathrm{u}-$ and $\mathrm{e}-$ Service, Science and Technology Vol.9, No. 3 (2016) 